\title{
Novel neuro-audiological findings and further evidence for TWNK involvement in Perrault syndrome
}

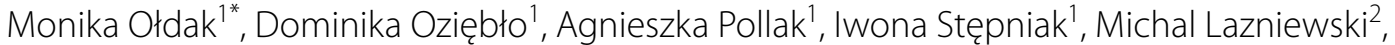 \\ Urszula Lechowicz ${ }^{1}$, Krzysztof Kochanek ${ }^{3}$, Mariusz Furmanek ${ }^{4}$, Grażyna Tacikowska ${ }^{5}$, Dariusz Plewczynski ${ }^{2}$, \\ Tomasz Wolak ${ }^{4}$, Rafał Płoski ${ }^{6}$ and Henryk Skarżyński ${ }^{7}$
}

\begin{abstract}
Background: Hearing loss and ovarian dysfunction are key features of Perrault syndrome (PRLTS) but the clinical and pathophysiological features of hearing impairment in PRLTS individuals have not been addressed. Mutations in one of five different genes HSD17B4, HARS2, LARS2, CLPP or TWNK (previous symbol C10orf2) cause the autosomal recessive disorder but they are found only in about half of the patients.

Methods: We report on two siblings with a clinical picture resembling a severe, neurological type of PRLTS. For an exhaustive characterisation of the phenotype neuroimaging with volumetric measurements and objective measures of cochlear hair cell and auditory nerve function (otoacustic emissions and auditory brainstem responses) were used. Whole exome sequencing was applied to identify the genetic cause of the disorder. Co-segregation of the detected mutations with the phenotype was confirmed by Sanger sequencing. In silico analysis including 3D protein structure modelling was used to predict the deleterious effects of the detected variants on protein function.

Results: We found two rare biallelic mutations in TWNK, encoding Twinkle, an essential mitochondrial helicase. Mutation c.1196A>G (p.Asn399Ser) recurred for the first time in a patient with PRLTS and the second mutation c.1802G $>A$ (p.Arg601GIn) was novel for the disorder. In both patients neuroimaging studies showed diminished cervical enlargement of the spinal cord and for the first time in PRLTS partial atrophy of the vestibulocochlear nerves and decreased grey and increased white matter volumes of the cerebellum. Morphological changes in the auditory nerves, their desynchronized activity and partial cochlear dysfunction underlay the complex mechanism of hearing impairment in the patients.
\end{abstract}

Conclusions: Our study unveils novel features on the phenotypic landscape of PRLTS and provides further evidence that the newly identified for PRLTS TWNK gene is involved in its pathogenesis.

Keywords: Perrault syndrome, TWNK, C10orf2, Whole exome sequencing, Auditory neuropathy, Hearing, Vestibulocochlear nerve

\section{Background}

Perrault syndrome (PRLTS) is suspected in a female with bilateral sensorineural hearing loss (SNHL), ovarian dysfunction that may occur in a mild (primary ovarian

\footnotetext{
*Correspondence: m.oldak@ifps.org.pl

1 Department of Genetics, World Hearing Center, Institute of Physiology and Pathology of Hearing, Mokra 17, Kajetany/Warsaw, 05-830 Nadarzyn, Poland

Full list of author information is available at the end of the article
}

insufficiency) or severe (ovarian dysgenesis) form and a normal 46,XX karyotype [1]. In males PRLTS is manifested by SNHL and their fertility is generally considered normal but this matter should be regarded with caution due to scarcity of males with PRLTS and data from the animal studies $[2,3]$. SNHL is usually the initial expression of PRLTS. In some patients a spectrum of neurologic symptoms develops over time indicating a progressive, age-related neurodegenerative process. 
Common neurological abnormalities are motor and sensory neuropathy, muscle weakness and atrophy, hypo- or areflexia, cerebellar ataxia, limited eye movements, nystagmus, dyspraxia, as well as intellectual deficit, developmental delay and seizures [4-7]. In brain magnetic resonance imaging (MRI), nonspecific white matter changes suggestive for cerebral leucodystrophy and cerebellar atrophy are described $[5,6,8]$. Depending on the occurrence of neurologic features, a classification of PRLTS into type I, static and without neurologic involvement and type II with a progressive neurological disease has been proposed [7].

Transmission of PRLTS is autosomal recessive and over the last years five genes causative for PRLTS have been identified. Mutations in HSD17B4 (OMIM *601860; PRLTS1), encoding a peroxisomal enzyme, were found in four PRLTS families [9-12]. Mutations in HARS2 (OMIM *600783; PRLTS2) were detected in three families [4, 13], mutations in LARS2 (OMIM "604544; PRLTS4) in six families [10, 13-15], mutations in CLPP (OMIM *601119; PRLTS3) in seven families [5, 10, 13, 16, 17], and mutations in TWNK (OMIM *606075; PRLTS5) only in four families with PRLTS $[6,10,13]$. All of the four latter PRLTS genes code for mitochondrial proteins. Molecular etiology for PRLTS remains unknown in as many as $55 \%$ of the patients, which emphasizes a genetic heterogeneity of the syndrome and shows that novel disease-causing genes still await discovery $[10,13]$.

Twinkle mtDNA helicase (TWNK), located in the long arm of chromosome 10 (10q24.31), encodes Twinkle, which localizes to mitochondrial nucleoids and forms a hexameric or heptameric ring structures. Twinkle is composed of a primase and helicase domain connected via a linker region, involved in stabilization of the oligomeric complexes of the protein. With the primase activity the protein initiates DNA replication and with the helicase activity unwinds DNA for replication. Twinkle is essential for the replication process of mitochondrial DNA (mtDNA) and lifetime maintenance of human mtDNA integrity $[18,19]$.

In this study, whole-exome sequencing (WES) was successfully applied to identify the molecular basis of PRLTS in the proband and her sister from a nonconsanguineous Polish family. The cardinal manifestations of PRLTS (SNHL and ovarian dysgenesis) in the patients were preceded and dominated by severe and progressive involvement of the nervous system, making the diagnosis not straightforward. Here, we provide a thorough neurological and audiological assessment of both patients that unveil novel features on the phenotypic landscape of PRLTS.

\section{Patients and methods}

\section{Study subjects}

Two affected sisters from a Polish non-consanguineous family and their unaffected mother were available for the study. The proband was born at term by Cesarean section after an uneventful pregnancy with $3600 \mathrm{~g}$ of body weight and an Apgar score of $1 / 2 / 3$, indicating severe birth depression. Her psychomotor development was normal until the third year of age, when the motor performance begun to deteriorate progressively and the electromyography (EMG) examination was abnormal (she is unable to run since the age of 15 years). Speech development was age appropriate. At 5 years of age SNHL was diagnosed and pathologic auditory brainstem responses (ABRs) were observed. She has been fitted with hearing aids at the age of 13 but they were of limited benefit. Testing for primary amenorrhea and delayed pubertal development at the age of 15 revealed hypergonadotropic hypogonadism, streak gonads, rudimentary uterus and a normal female karyotype 46,XX. Hormone replacement therapy was introduced. At the age of 16 chronic thyroiditis with elevated levels of anti-thyroid peroxidase antibodies was diagnosed. Nerve conduction studies at the age of 21 showed axonal sensorimotor polyneuropathy. Ophthalmological examination was unremarkable except for impaired eye movements (Table 1).

The proband's sister was born at term with $2850 \mathrm{~g}$ of body weight and an Apgar score of 5/6/7. Horizontal nystagmus and imbalance began at the age of 11, walking was gradually deteriorating and sensorimotor polyneuropathy was identified. At the age of 12 SNHL and ovarian dysgenesis with normal female karyotype were diagnosed. From the age of 16 years she has a Hashimoto's disease. Biochemical studies showed mildly elevated levels of serum lactate and creatine kinase. Metabolic disease screening for organic acids with gas chromatography-mass spectrometry (GS/MS) in urine, amino acids and acylcarnitines with liquid chromatography-tandem mass spectrometry (LC-MS/MS) in dried blood spot and congenital disorders of glycosylation gave normal results. Wilson and Refsum diseases were excluded based on normal blood concentrations of ceruloplasmin and cooper and phytanic acid, respectively.

\section{Neurological and audiological evaluation}

Written informed consent was obtained from each participant. The study was approved by the ethics committee at the Institute of Physiology and Pathology of Hearing and performed according to the Declaration of Helsinki. The patients underwent thorough clinical evaluation. Functional impairment was assessed according to spinocerebellar degeneration functional score (SDFS) [20]. In brain and cervical spine MRI (3T Siemens Magnetom 
Table 1 Clinical features and laboratory findings in the affected family members

\begin{tabular}{|c|c|c|}
\hline Clinical features & Proband & Sister \\
\hline Sex & $\mathrm{F}$ & $\mathrm{F}$ \\
\hline Age at disease onset, years & 3 & 11 \\
\hline Age at examination, years & 27 & 19 \\
\hline Disease duration & 24 & 8 \\
\hline Sensorineural hearing loss & $+\left(5^{*}\right)$ & $+\left(12^{*}\right)$ \\
\hline Ovarian dysfunction & $+\left(15^{*}\right)$ & $+\left(12^{*}\right)$ \\
\hline Intellectual disability & - & - \\
\hline Dementia & - & - \\
\hline Epilepsy & - & - \\
\hline Cerebellar syndrome & $+\left(3^{*}\right)$ & $+\left(11^{*}\right)$ \\
\hline SDFS & 3 & 2 \\
\hline Impaired eyes movement & + & + \\
\hline $\begin{array}{l}\text { Gaze-evoked horizontal } \\
\text { nystagmus }\end{array}$ & + & + \\
\hline $\begin{array}{l}\text { Gaze-evoked vertical nystag- } \\
\text { mus }\end{array}$ & - & + \\
\hline Dysarthria & + & + \\
\hline Ataxia & + & + \\
\hline Positive Romberg's test & + & + \\
\hline Flaccid paresis & $+\left(?^{*}\right)$ & $+\left(?^{*}\right)$ \\
\hline Muscle weakness & $+\mathrm{UL}<\mathrm{LL}$ & $+\mathrm{UL}<\mathrm{LL}$ \\
\hline Muscle atrophy & $+\mathrm{UL}<\mathrm{LL}$ & $+\mathrm{UL}<\mathrm{LL}$ \\
\hline Tendon reflexes UL & Diminished & Diminished \\
\hline Tendon reflexes LL & Absent & Absent \\
\hline Gait & Steppage & Steppage \\
\hline High-arched palate & + & + \\
\hline Pes cavus and clawed toes & + & + \\
\hline $\begin{array}{l}\text { Other features_-Hashimoto } \\
\text { disease }\end{array}$ & + & + \\
\hline \multicolumn{3}{|l|}{ Laboratory findings } \\
\hline Lactate elevation & n.a. & + \\
\hline CK elevation & n.a. & + \\
\hline FSH and LH elevation & + & + \\
\hline EMG & $\begin{array}{l}\text { Axonal, sensori- } \\
\text { motor polyneu- } \\
\text { ropathy }\end{array}$ & $\begin{array}{l}\text { Axonal, sensori- } \\
\text { motor polyneu- } \\
\text { ropathy }\end{array}$ \\
\hline Abnormal neuroimaging & + & + \\
\hline
\end{tabular}

Numbers in brackets (*) refer to age at diagnosis, years

UL upper limbs, LL lower limbs, n.a. no data available

Trio, 12-channel Head Matrix Coil) T1-weighted, T2-weighted and diffusion-weighted images were acquired. In addition, high-resolution 3D structural T1-weighted volumes were acquired using an MPRAGE sequence with 208 sagittal slices and an isotropic resolution $0.9 \times 0.9 \times 0.9 \mathrm{~mm}$. Sequence parameters were:
$\mathrm{TR}=1900 \mathrm{~ms}, \mathrm{TE}=2.21 \mathrm{~ms}, \mathrm{TI}=900 \mathrm{~ms}, \mathrm{FA}=9$, FOV $=26 \times 28.8 \mathrm{~cm}$, matrix $=320 \times 290$, Pixel bandwidth $=200 \mathrm{~Hz} /$ pix, iPAT $=2$, TA $=5 \mathrm{~min}$. Brain structures were segmented by Freesurfer version 5.3 (http:// surfer.nmr.mgh.harvard.edu/). The diameter of the vestibulocochlear nerve was evaluated directly and compared with the neighboring facial nerve used as an internal reference. Assessment of the cochlear and vestibular components was based on their visual comparison and with reference to the facial nerve. For comparisons of the cerebrum and cerebellum volumes and their white and gray matters, age- and sex-matched controls [21-23] from the Internet Brain Volume Database funded by The Human Brain Project (http://ibvd.virtualbrain.org/) were used. A difference above 2.3 of the standard deviation was considered as statistically significant.

Assessment of auditory function consisted of pure-tone and speech audiometry, impedance audiometry, otoacoustic emissions (OAE) and ABRs. Hearing thresholds for air and bone conduction were determined at frequencies $125-8000$ and $250-4000 \mathrm{~Hz}$, respectively, using the AC40 clinical audiometer (Interacoustics, Middelfart, Denmark) and the 10/5 dB descending-ascending threshold estimation procedure [24]. Speech comprehension was tested using monosyllabic Polish words and the AC40 audiometer (Interacoustics) [25]. Acoustic impedance measurements (tympanograms and stapedius reflex) were performed with the Zodiac 901 instrument (Madsen Electronics, Copenhagen, Denmark). Stapedius reflexes were analyzed for the frequencies 500, 1000, 2000 and $4000 \mathrm{~Hz}$ in the ipsi and contralateral modes [26]. OAE were evoked by standard-click stimuli and $500 \mathrm{~Hz}$ tone bursts by using the ILO-292 system (Otodynamics Ltd, Hatfield, United Kingdom) [27-29]. ABRs were recorded using the Integrity V500 system (Vivosonic Inc., Toronto, Canada). The stimuli were $0.1 \mathrm{~ms}$ clicks with alternating polarity presented with $90 \mathrm{~dB}$ normal hearing level (nHL) intensity at a repetition rate of $11 / \mathrm{s}$. The amplifier bandwidth was $30-1500 \mathrm{~Hz}$ and analysis time $12 \mathrm{~ms}$. The number of sweeps required for an averaged response was 1024 [30].

For evaluation of the vestibular endorgan function Fitzgerald and Hallpike bithermal caloric test with video eye movement recordings (Visual Eyes Micromedical Technologies, Chatham, USA) were used. Sinusoidal harmonic acceleration testing at frequencies $0.01-0.32 \mathrm{~Hz}$ was conducted with a Rotational Vestibular Chair System 2000 (Micromedical Technologies, Chatham, USA). Otolith function was measured using air-conducted sound stimulation cervical and ocular vestibular evoked myogenic potentials (cVEMP, oVEMP) at $500 \mathrm{~Hz}, 95 \mathrm{dBnHL}$ (EclipsVemp, Interacoustics, Assens, Denmark). 


\section{Whole-exome and Sanger sequencing}

DNA was isolated from blood sample by a standard procedure. WES was performed using SureSelect Target Enrichment (Agilent Technologies, Palo Alto, CA, USA) according to the manufacturer's protocol. The sample was run on $16 \%$ of a lane on HiSeq 1500 using $2 \times 100 \mathrm{bp}$ paired-end reads. All bioinformatics analysis was done as described previously [31]. After primarily CASAVA processing, all reads were aligned to the hg19 reference genome with the Burrows-Wheeler Alignment Tool and analyzed with Genome Analysis Toolkit [32]. Indel realignment, base quality score recalibration, duplicates elimination as well as SNP/INDEL calling were performed [33]. The retrieved variants were annotated with ANNOVAR and converted to MS Access format for subsequent manual analyses. Total exon coverage by 20 reads or more was $89 \%$ and by 10 reads or more $95.9 \%$. Alignments were inspected with Integrative Genomics Viewer [34] and analyzed with a pipeline combining protein coding changes, splice site prediction, prevalence in populations, evolutionary conservation and scores from PolyPhen-2 [35], SIFT [36] and MutationTaster2 [37] prediction algorithms of non-synonymous single-nucleotide variants. Sanger sequencing with $3500 x \mathrm{~L}$ Genetic Analyzer (Applied Biosystems, Foster City, CA, USA) and BigDye Terminator cycle sequencing kit v. 3.1 (Applied Biosystems) were used to confirm the presence of variants identified by WES.

\section{In silico protein analysis}

Homologs of the human Twinkle protein were identified with a PSI-Blast [38] search (E-value threshold of 0.005 ) performed against the NCBI non-redundant protein sequence database. The collected 15,000 sequences were initially clustered at $60 \%$ sequence identity with cdhit [39] and any sequences shorter than 300, longer than 1000 amino acids or described as "hypothetical protein" were removed. The multiple sequence alignment of the twinkle family was derived using MAFFT program [40].

For preparation of the Twinkle homology model, the crystal structure of the gp4 protein from bacteriophage T7 (Protein Data Bank code1e0j) was selected as a template after analyzing the GeneSilico Metaserver results [41]. The sequence-to-structure alignment between the Twinkle protein and the template (Additional file 1: Figure S1) was built using the consensus alignment approach and 3D assessment [42] based on the results of FFAS [43], HHSearch [44] and the alignment proposed by Fernandez et al. [19]. Multiple sequence alignment of the family was also taken into consideration. The 3D model of the protein was built with MODELLER [45]. A model quality assessment was carried out using ProSA-web server [46]. Secondary structure elements were predicted with
PSI-PRED [47]. Structure visualization was carried out with PyMOL (http://www.pymol.org).

\section{Results \\ Involvement of the nervous system}

The proband and her sister came to our observation at the age of 27 and 19, respectively. They both had progressive neurologic symptoms, including postlingual progressive SNHL, cerebellar syndrome and flaccid paresis (Table 1) with muscle atrophy being more prominent in the proband.

Brain MRI in both sisters revealed considerable bilateral thinning of the vestibulocochlear nerve and its cochlear and vestibular components, which was defined as partial atrophy. It was more pronounced in the cochlear than in the vestibular nerves. Morphological signs of a subtle cerebellar atrophy (widening of the sulci more pronounced in the vermis than in hemispheres) were found in the proband but not in the sister. In cervical spine MRI, a diminished cervical enlargement was observed in both patients (Fig. 1). In the proband volumetric measurements of the brain showed significantly increased cerebellum white and decreased cerebellum gray matter. The results of other volumetric measurements were unchanged (Table 2).

\section{Functional alterations in vestibulocochlear pathway}

In the proband and her sister, a different degree of sensorineural hearing loss mainly affecting high frequencies has been diagnosed in pure-tone audiometry (Fig. 2a). In contrast to pure-tone thresholds, speech discrimination was unproportionally poor and the test has not been continued. In both patients, the tympanograms revealed normal middle ear function. Ipsi- and contralateral acoustic reflexes were absent for all tested frequencies. The analysis of OAE recordings from both patients showed the presence of otoacoustic emission signals in the frequency range up to $2 \mathrm{kHz}$ in the right ear and up to $4 \mathrm{kHz}$ in the left ear. OAE in both patients were largely consistent with the results of pure-tone audiometry, demonstrating a partially impaired function of the outer hair cells. In ABR recordings, no responses at the maximum level of $90 \mathrm{~dB}$ nHL were obtained biaurally in the proband (Fig. 2b) and her sister. Comprehensive audiological evaluation revealed auditory neuropathy that was accompanied by a certain degree of cochlear dysfunction.

In both patients bi-thermal caloric irrigation results were within the normal limit. Rotational testing showed abnormally reduced gain and increased phase at 0.01 , 0.02 and $0.04 \mathrm{~Hz}$ and was unaffected at the remaining frequencies. In the proband cVEMP and oVEMP were not recorded on either side. In her sister only oVEMP on the left side was registered with a prolonged latency of N1 and P1 peaks (data not shown). The results are 

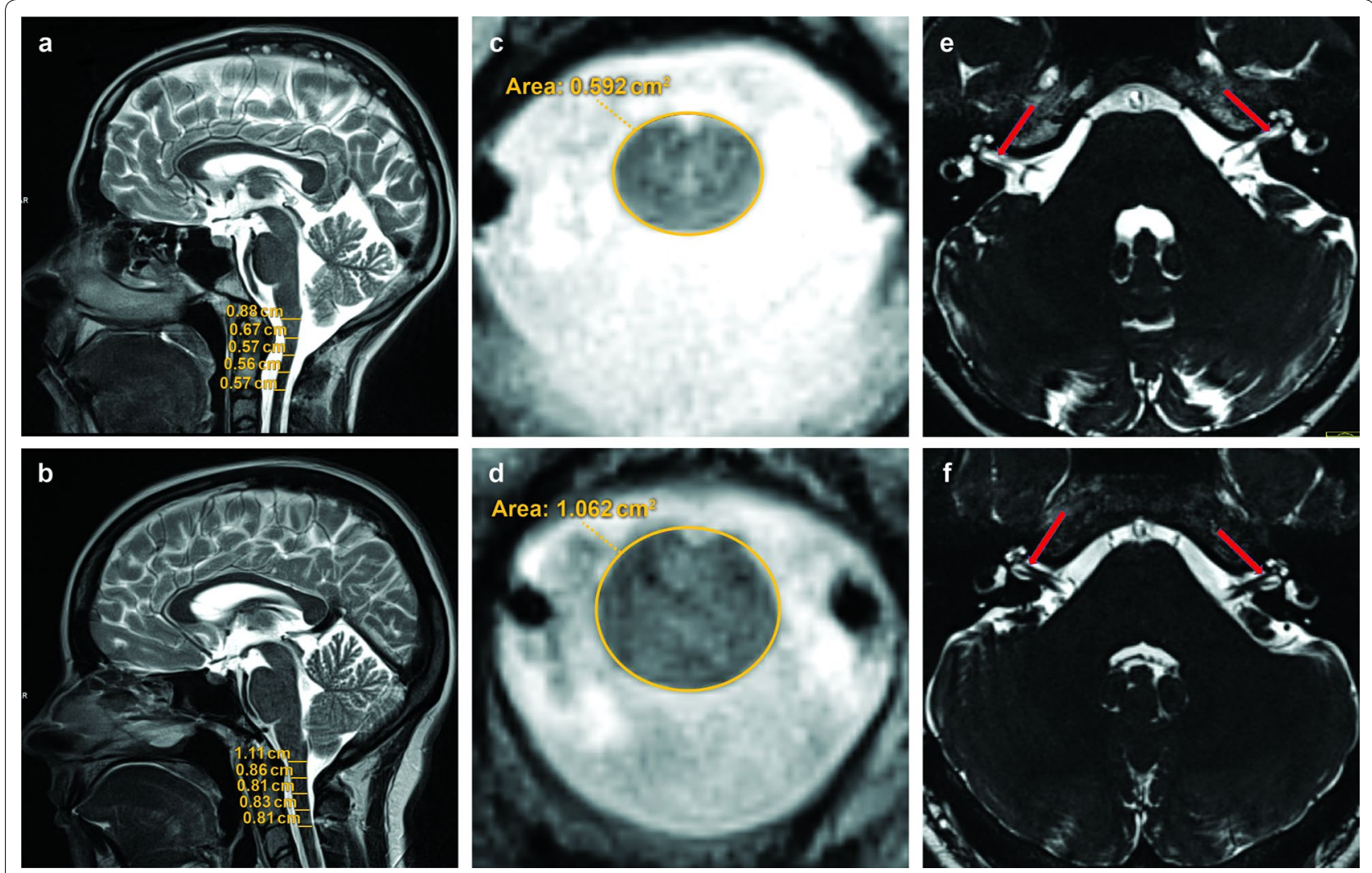

Fig. 1 Brain and spine MRI in the proband. The left column shows sagittal T2 weighted images of the head and cervical spine (a proband, $\mathbf{b}$ control) with the measurements of spinal cord thickness at different levels. The middle column shows cross-section of the spinal cord (c proband, $\mathbf{d}$ control). The right column shows transverse heavy $\mathrm{T} 2$ weighted images of sub-millimeter slice thickness displaying the cochlear nerve, pointed with a red arrow (e proband, $\mathbf{f}$ control)

Table 2 Volumes of cerebrum, cerebellum and the respective gray and white matters in the proband and her sister

\begin{tabular}{|c|c|c|c|c|}
\hline Brain part & Proband $\left(\mathrm{cm}^{3}\right)$ & Control groups $\left(\mathrm{cm}^{3}\right)^{\mathrm{a}}$ & Proband's sister $\left(\mathrm{cm}^{3}\right)$ & $\begin{array}{l}\text { Control } \\
\text { group } \\
\text { b }\left(\mathrm{cm}^{3}\right)\end{array}$ \\
\hline \multirow[t]{2}{*}{ Cerebrum volume } & 1030 & $1181.3 \pm 83.1$ & 1187.1 & $1172 \pm 108.1$ \\
\hline & & $1148.5 \pm 77.2$ & & \\
\hline \multirow[t]{2}{*}{ Cerebrum gray matter volume } & 571 & $672.3 \pm 33.3$ & 674.2 & $579 \pm 50.6$ \\
\hline & & $663.2 \pm 51$ & & \\
\hline \multirow[t]{2}{*}{ Cerebrum white matter volume } & 406 & $384.6 \pm 40.5$ & 464.2 & $435 \pm 50.6$ \\
\hline & & $425.3 \pm 30$ & & \\
\hline \multirow[t]{2}{*}{ Cerebellum volume } & 131.1 & $153.8 \pm 10.8$ & 145.2 & $142 \pm 17.5$ \\
\hline & & $134.7 \pm 6.8$ & & \\
\hline \multirow[t]{2}{*}{ Cerebellar gray matter volume } & $\underline{98.4}$ & $128.1 \pm 9.2$ & 111.9 & n.a. \\
\hline & & $112.3 \pm 5.9$ & & \\
\hline \multirow[t]{2}{*}{ Cerebellar white matter volume } & $\underline{32.7}$ & $26 \pm 2.2$ & 33.7 & n.a. \\
\hline & & $22.4 \pm 2$ & & \\
\hline
\end{tabular}

n.a. no data available

a Control groups from two different studies: first values [21], second values [22]

b Control group from [23]; underlined are statistically significant differences from both control groups 
a

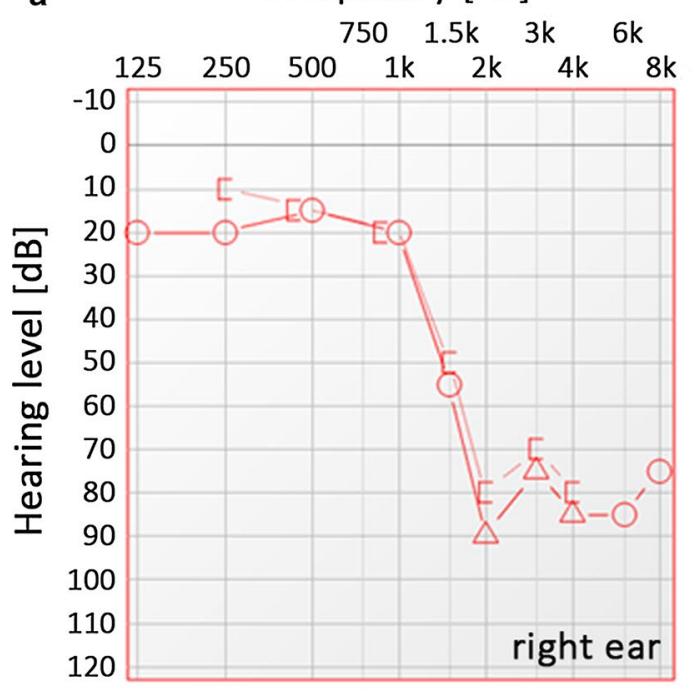

\section{Frequency $[\mathrm{Hz}]$}

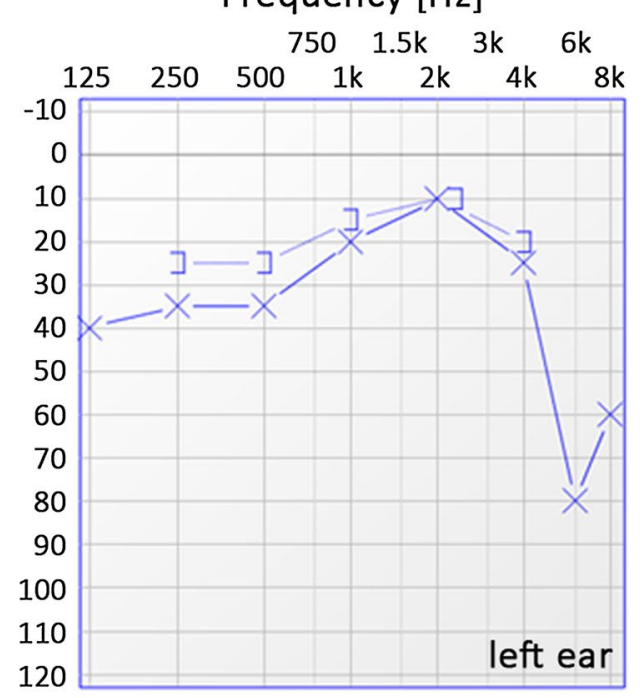

b

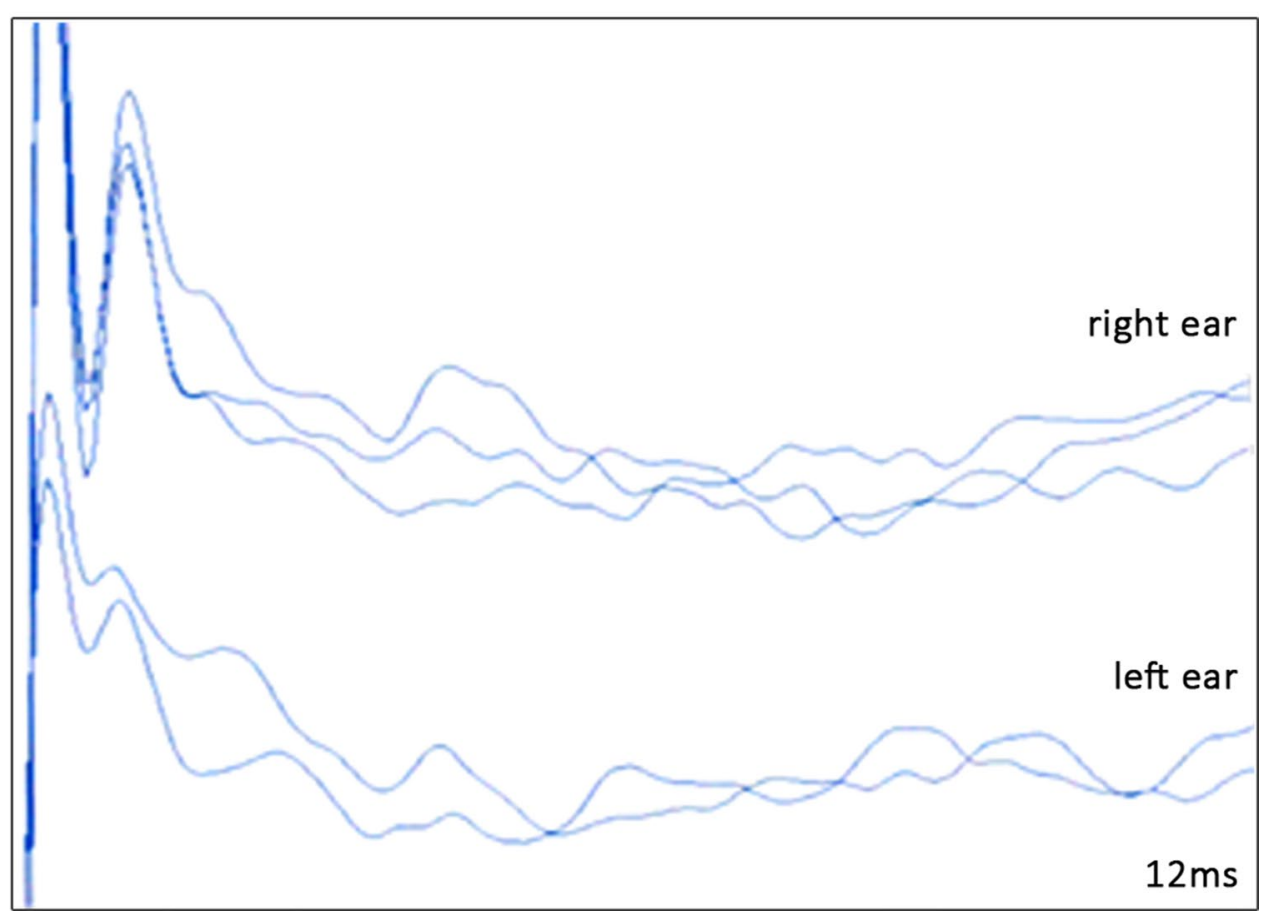

Fig. 2 Pure tone audiometry (a) and ABRs (b) of the proband. a " $O$ " and " $X$ " symbols denote air conduction thresholds in the right and left ear, respectively and " $\triangle$ " denotes masked air conduction at high frequencies in the right ear; "[" or "]" denote masked bone conduction. b Shown are ABR recordings after click stimulus at an acoustic level of $90 \mathrm{~dB}$ normal hearing level $(\mathrm{nHL})$ and presentation rate of 11/s

suggestive of a neuropathy of both the superior and the inferior vestibular nerves in addition to the involvement of the auditory branch of the vestibulocochlear nerve.

Identification of compound heterozygous TWNK mutation DNA sample of the proband was analyzed by whole exome sequencing. After exclusion of variants found with a prevalence of $1 \%$ or more in the databases of the Exome Aggregation Consortium (ExAC, http://exac. broadinstitute.org/), 1000 Genomes Project (http:// www.1000genomes.org) and the NHLBI GO Exome Sequencing Project (ESP, http://evs.gs.washington.edu/ EVS/; all accessed 05/2016) and in a set of 816 exomes of Polish patients (ZGM, R. Płoski, unpublished results) 
in the first line we searched for variants reported in the Human Gene Mutation Database (www.hgmd.cf.ac.uk/ ac/index.php) and variants predicted to be pathogenic by bioinformatic tools. We found a rare TWNK heterozygous missense variant NM_021830.4:c.1196A>G (rs863223921), causing the missense change NP_068602.2:p.Asn399Ser (Fig. 3a, b) that has been identified for the first time in 2016 in a Norwegian female with PRLTS [10] (Table 3). The variant is predicted to be damaging by PolyPhen-2 (score 0.993), SIFT (score 0.02) and MutationTaster2 (score 0.998).

The second $T W N K$ variant was a very rare heterozygous missense change NM_021830.4:c.1802G>A (rs141315771), causing the amino acid substitution NP_068602.2:p.Arg601Gln (Fig. 3a, b). The p.Arg601Gln variant was reported only in the ExAC and ESP databases (accessed 07/2016) with an allele frequency of $3.29 \mathrm{e}-5$ (4/121412 alleles) and 1.54e-4 (2/13006 alleles), respectively but heretofore it has not been identified in a homozygous state or associated with any disease. The G>A transition is predicted to be damaging by PolyPhen-2 (score 0.895), SIFT (score 0.02) and MutationTaster2 (score 0.997).

Presence of the two heterozygous TWNK mutations was confirmed in the proband's sister. The mother was a carrier of the p.Asn399Ser mutation, showing that the two TWNK mutations are biallelic and the patients are compound heterozygous for the mutations (Fig. 3a, b). DNA sample from the deceased father was not available for the study.

\section{Modelling of p.Asn399Ser and p.Arg601Gln functional roles}

Multiple sequence alignment demonstrated that Asn399 and Arg601 are conserved amino acid residues among vertebrates (Fig. 4a). In the crystal structure of the bacteriophage T7 gp4 protein (human Twinkle homolog), Asn289 (human Asn399) forms two hydrogen bonds with the backbone of Phe296 (human Trp392). A similar scenario is observed for the human protein. The side chain of Asn399, which is located on the short helix, forms two hydrogen bonds with the backbone atoms of Trp392. Both amino acids are located in a region between the linker and helicase domains. The p.Asn399Ser mutation disrupts these interactions as Ser399 is located too far away to form hydrogen bonds with the main chain of Trp392 (Fig. 4b, c). As a result, the entire region may adopt a different conformation than in the wild type protein. This may impair the correct orientation of the linker region and hinder the hexamer/heptamer formation [19].
In the $\mathrm{T} 7 \mathrm{gp} 4$ protein the adenine ring of the ATP analog is stacked between Arg504 (human Asp591) and Tyr535 (human Phe621) [48]. Val514 (human Arg601) is positioned next to the adenine ring and placed on the same plane. In the human Twinkle protein the key Arg504 is replaced by an aspartic acid, which is unable to stabilize the adenine ring. Simultaneously, an arginine (Arg601) is introduced at the position occupied by a short-chained amino acid (Val514) in the T7 phage. According to our 3D model, human Arg601 could adopt a conformation, which allows the formation of a hydrogen bond with the ATP N7 atom. This conformation can be stabilized by hydrogen bond interactions with Asp591. In contrast to the T7 phage Arg504, human Arg601 seems not to form a cation- $\pi$ interaction with the adenine ring. However, we cannot exclude the possibility that the loop, on which both Arg601 and Asp591 are located, assumes a different conformation than in our model. This may allow Arg601 to adopt a conformation where the adenine ring is stabilized via stacking rather than hydrogen bonding. The interaction between ATP and Phe621 remains unaffected. Substitution of Arg601 to Gln most likely weakens the binding of ATP as Gln is unable to form a hydrogen bond with the adenine N7 atom (Fig. 4d, e).

\section{Discussion}

Herein, we report the identification of a distinctive phenotype of PRLTS5 (OMIM \#616138), in which the progressive neurologic features, dominating in the phenotype, preceded the diagnosis of SNHL and ovarian dysfunction. Comprehensive analysis of the patients' phenotype and family history enabled us to establish a clinical suspicion of PRLTS. After applying WES the underlying cause of the disorder has been explained by the detection of compound heterozygous mutations in the TWNK. It is the most recently discovered gene involved in the pathogenesis of PRLTS that heretofore has been reported only in four PRLTS families worldwide.

Pathogenic role of heterozygous TWNK mutations have been first discovered in families with autosomal dominant progressive external ophthalmoplegia (PEOA3; OMIM \#609286) [18]. Recessive TWNK mutations are causative for mitochondrial DNA depletion syndrome 7 (MTDPS7; OMIM \#271245) also known as infantile-onset spinocerebellar ataxia (IOSCA) $[49,50]$ and were recently identified in patients with PRLTS5 $[6,10]$ (Table 3). There is a substantial phenotypic overlap across the conditions resulting from TWNK mutations, particularly in regard to the neurological features. Hearing loss, ataxia, myopathy, neuropathy and ophthalmoplegia have been reported in patients with each of these diseases. In our patients the diagnosis of PEOA3 could be excluded based on the presence of ovarian dysgenesis that has not 
a
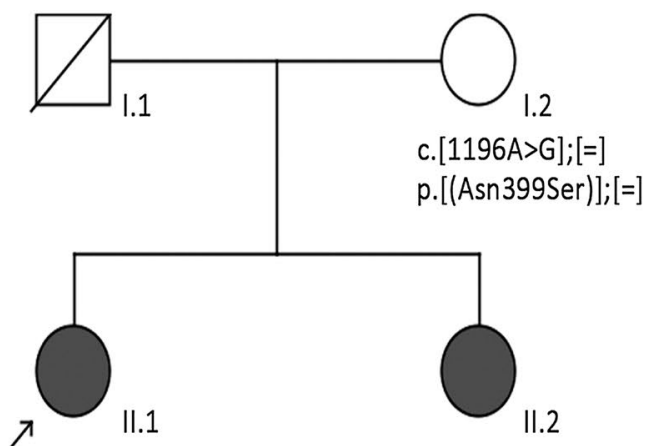

c. $[1196 \mathrm{~A}>\mathrm{G}] ;[1802 \mathrm{G}>\mathrm{A}]$
p.[(Asn399Ser) $) ;[(\operatorname{Arg} 601 \mathrm{GIn})]$

b
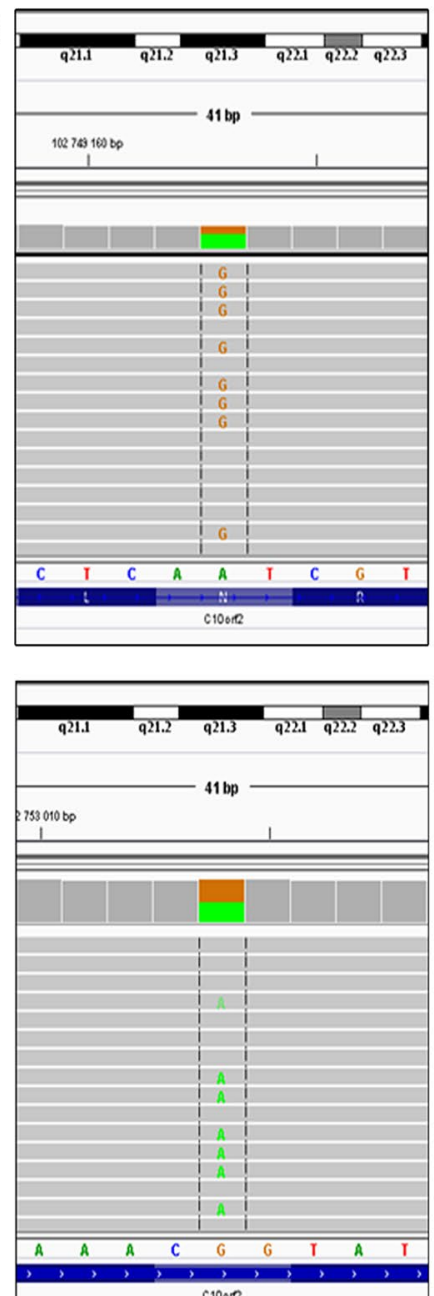

$6100 \mathrm{rg}$ c. [1196A>G];[1802G>A]

p. [(Asn399Ser) $] ;[(\operatorname{Arg} 601 \mathrm{G} \ln )]$
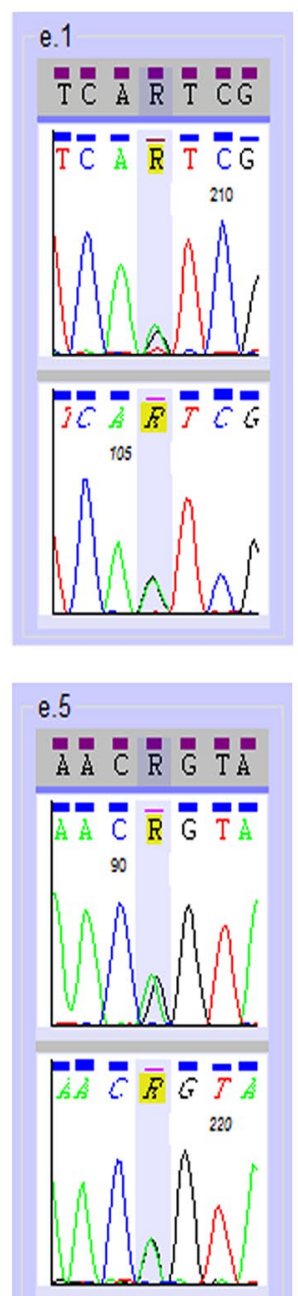

Fig. 3 Identification of TWNK mutations in the analyzed families. a Pedigree of the investigated family. The proband is marked with an arrow. Black symbols indicate individuals affected with PRLTS5 and open symbols indicate unaffected individuals; diagonal line denotes the deceased father. The TWNK genotypes identified in the family members are reported at the cDNA and protein levels according to the HGVS-nomenclature (http:// varnomen.hgvs.org/; accessed 07/2016). b WES in the proband revealed an A>G transition (upper left panel) and G>A transition (lower left panel), corresponding to p.Asn399Ser (AAT>AGT) and p.Arg601Gln (CGG>CAG), respectively, in the TWNK gene. Direct Sanger sequencing of TWNK confirmed the presence of the two mutations (right panel). For each mutation sequencing of the forward (top) and the reverse (bottom) strand is shown 
Table 3 Comparison of demographic and molecular findings in PRLTS patients with TWNK mutation

\begin{tabular}{|c|c|c|c|c|c|c|c|}
\hline $\begin{array}{l}\text { Family \# and } \\
\text { origin }\end{array}$ & Consanguinity & PRLTS & $\begin{array}{l}\text { Reference } \\
\text { sequence number }\end{array}$ & $\begin{array}{l}\text { Mutation cDNA } \\
\text { level }\end{array}$ & $\begin{array}{l}\text { Mutation protein } \\
\text { level }\end{array}$ & Karyotype & Reference \\
\hline \multirow[t]{2}{*}{ 1. Japanese } & \multirow[t]{2}{*}{ N } & \multirow[t]{2}{*}{ Two sisters } & rs556445621 & c.1172G>A & p.Arg391His & \multirow[t]{2}{*}{$46, X X$} & \multirow[t]{2}{*}[6]{} \\
\hline & & & rs672601360 & c. $1754 A>G$ & p.Asn585Ser & & \\
\hline \multirow{2}{*}{$\begin{array}{l}\text { 2. American } \\
\text { with European } \\
\text { ancestry }\end{array}$} & \multirow[t]{2}{*}{$\mathrm{N}$} & \multirow[t]{2}{*}{ Two sisters } & rs672601361 & c. $1321 \mathrm{~T}>\mathrm{G}$ & p.Trp441Gly & \multirow[t]{2}{*}{$46, X X$} & \multirow[t]{2}{*}[6]{} \\
\hline & & & rs369588002 & C.1519G>A & p.Val507lle & & \\
\hline \multirow[t]{2}{*}{ 3. Norwegian } & \multirow[t]{2}{*}{ N } & \multirow[t]{2}{*}{ One female } & rs770917763 & c.968G>A & p.Arg323GIn & \multirow[t]{2}{*}{$46, X X$} & \multirow[t]{2}{*}{ [10] } \\
\hline & & & rs863223921 & c.1196A>G & p.Asn399Ser & & \\
\hline \multirow[t]{2}{*}{ 4. Moroccan } & \multirow[t]{2}{*}{ Y } & \multirow{2}{*}{$\begin{array}{l}\text { Two sisters and } \\
\text { brother }\end{array}$} & rs764669712 & c.793C>T & p.Arg265Cys & \multirow[t]{2}{*}{ n.a. } & \multirow[t]{2}{*}{ [13] } \\
\hline & & & rs764669712 & c.793C>T & p.Arg265Cys & & \\
\hline \multirow[t]{2}{*}{ 5. Polish } & \multirow[t]{2}{*}{$N$} & \multirow[t]{2}{*}{ Two sisters } & rs863223921 & c.1196A>G & p.Asn399Ser & \multirow[t]{2}{*}{$46, X X$} & \multirow[t]{2}{*}{ Present study } \\
\hline & & & rs141315771 & c. $1802 \mathrm{G}>\mathrm{A}$ & p.Arg601Gln & & \\
\hline
\end{tabular}

N no, $Y$ yes, n.a. no data available

been described in patients with PEOA3, an earlier age of disease onset (1-2 vs. 2-8 decade of life) and the identification of two TWNK mutations that were not pathogenic in the patients' parents (heterozygous carriers). In contrast, MTDPS7 begins very early in life, in children below 2 years of age; the course of the disease is severe and includes optic atrophy, intellectual disability and hepatic involvement that were not observed in our patients.

All mutations found in TWNK are missense changes and their location does not explain different clinical manifestations. It has been hypothesized that even slight disturbances to the Twinkle protein, as a consequence of TWNK mutation, may affect its enzymatic activity, DNA binding ability, interaction with subunits or stability and result in a less-effective enzyme [51, 52]. Different bioinformatics tools predicted a deleterious effect of p.Asn399Ser and p.Arg601Gln on the protein function. In the applied 3D model we showed that p.Asn399Ser may affect the oligomeric Twinkle structure, crucial for the enzyme's ability to unwind the DNA. A consequence of p.Arg601Gln appears to be impaired binding and hydrolysis of ATP, which is of paramount importance for enzyme functioning.

No formal criteria have been elaborated to facilitate accurate recognition of PRLTS. In the first line, karyotype analysis should be performed to exclude Turner syndrome or other abnormalities of the $\mathrm{X}$ chromosome as approximately half of females with Turner syndrome (gonadal dysgenesis) suffer from hearing loss [53]. Next, other causes of sensorineural hearing loss (SNHL) and ovarian dysfunction should be considered. Both conditions are genetically heterogeneous and testing of causative mutations in known genes is appropriate. In patients with neurologic involvement, the phenotype of PRLTS may overlap clinically with a mild form of peroxisomal D-bifunctional protein deficiency (DBP type IV; OMIM
\#261515). Presence of ovarian dysgenesis is considered the major clinical feature differentiating PRLTS from DBP type IV [9]. A comprehensive analysis of WES data provided us with a wealth of information on the genetic constitution of the proband. Except for two TWNK mutations no other mutation has been identified, which alone or in combination with other pathogenic variants could account for the clinical features observed in the proband. After identifying both TWNK mutations in the proband's sister and confirming their biallelic status we could unequivocally establish the molecular genetic basis of the disease in the studied family. Our results provide further evidence that mutations in TWNK cause PRLTS5 $[6,10]$.

Neurological features in PRLTS have been observed in patients with mutations either in HSD17B4, CLPP or TWNK genes, i.e. in three out of five known PRLTS genes $[5,6,9-12,16,17]$. However, involvement of the nervous system seems to be a constant finding only in patients with $T W N K$ mutations. In contrast to our patients in other individuals with PRLTS5 the neurological problems became noticeable later in life after hearing loss and ovarian dysfunction have been diagnosed $[6,10$, 13]. The proband had a subtle atrophy of the cerebellum, a feature previously described in PRLTS patients $[9,10]$. Significantly distorted proportion between the cerebellum white and grey matters represents a novel finding in PRLTS patients, which was independently confirmed using two different control groups. Atrophy of cervical medulla, present in both of our patients, was a feature of another PRLTS5 patient, who also shared the TWNK p.Asn399Ser mutation [10].

Audiological examination and neuroimaging studies revealed that hearing loss in PRLTS5 patients has a complex background. Analysis of the hearing threshold together with OAE (absent at higher frequencies) pointed 


\begin{tabular}{|c|c|c|c|}
\hline \multicolumn{4}{|l|}{ a } \\
\hline & Region I & Region II & Region III \\
\hline & $392 \quad 399$ & $591 \quad 601$ & 621 \\
\hline >Homo sapiens & AGLRWSRFPDLNRILKGH & LQDRKL ( 4 ) GKRY & LEFNK \\
\hline > Macaca mulatta & AGLRWSRFPDLNRILKGH & LQDRKL ( 4 ) GKRY & LEFNK \\
\hline >Equus caballus & AGIRWGRFPDLNRLLKGH & LQDRKL ( 4 ) GKRY & LEFNK \\
\hline > Rattus norvegicus & AGVRWSRFPDLNRLLKGH & LQDRKL ( 4 ) GKRY & LEFNK \\
\hline >Mus musculus & AGVRWSRFPDLNRLLKGH & LQDRKL ( 4 ) GKRY & LEFNK \\
\hline$>$ Gallus gallus & AGVKWARFPELNKLLKGH & LQDRKL ( 4 ) GKRY & LEFSK \\
\hline >Danio rerio & AGIQWSRFPELNRILKGH & LQEKKL ( 4 ) GRRS & LEFNK \\
\hline >orcinus orca & AGVRWSRFPDLNRLLKGH & LQDRRL ( 4 ) GKRY & LEFNK \\
\hline
\end{tabular}
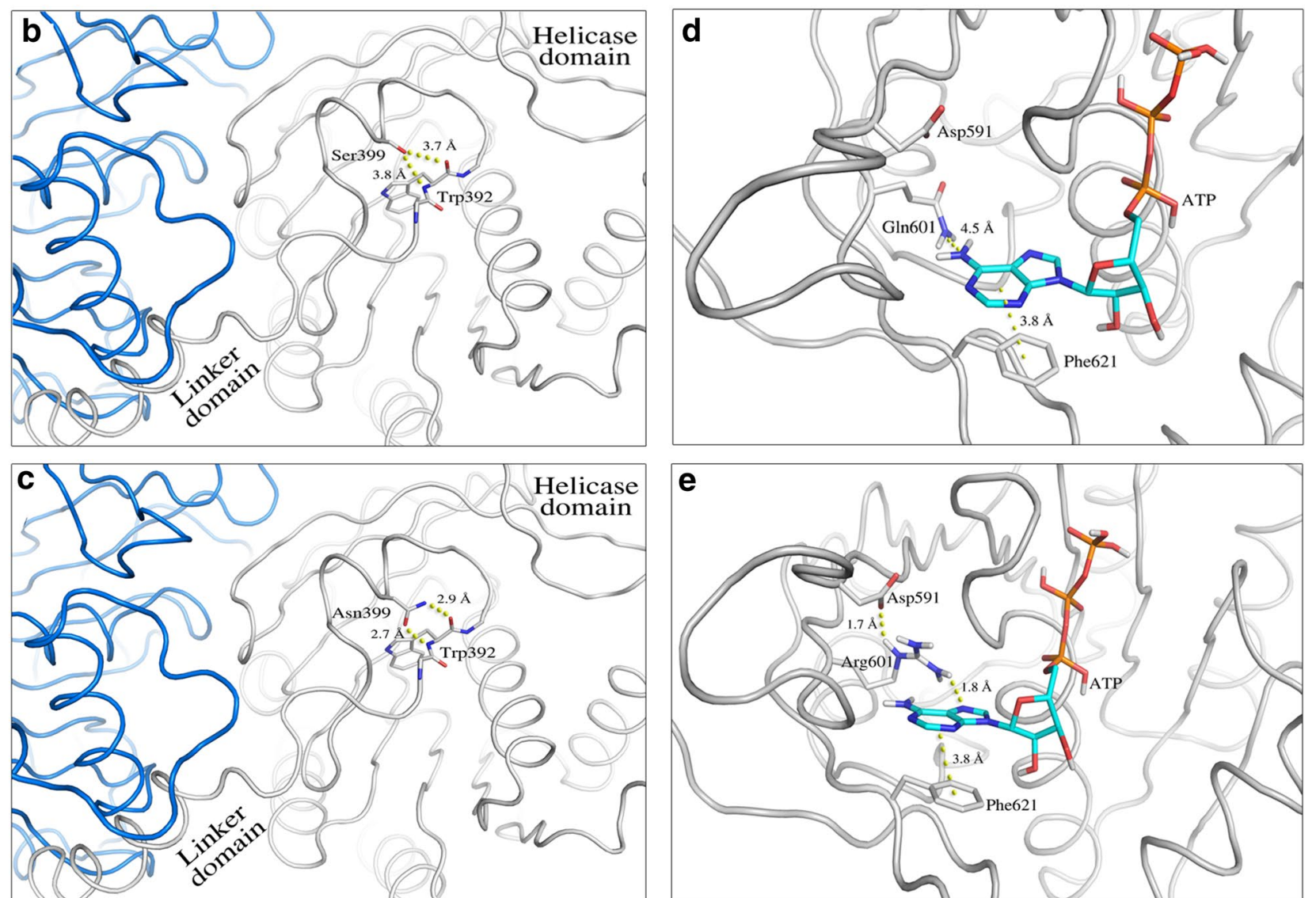

Fig. 4 Multiple protein sequence alignment and 3D structure of the human Twinkle protein in regions encompassing p.Asn399Ser and p.Arg601Gln mutations. a Multiple protein sequence alignment of selected sequences. Two regions of the twinkle protein are shown: (i) the region joining the linker and the helicase domains (Region I) and (ii) the region involved in stabilizing the adenine ring of ATP (Regions I/ and III). The numbers above the alignment correspond to the amino acid position in the human protein sequence. (b-c) Two interacting monomers of the Twinkle protein are colored white and blue. Changes in the conformation of a region next to the linker domain in p.Asn399Ser mutant (b) and in wild type (c) proteins are shown. (d-e) Changes in hydrogen bonds network resulting in weakened ATP binding in the p.Arg601Gln mutant (d) as compared to wild type (e) protein are depicted 
to impairment of the cochlear function. While lack of stapedius reflexes and ABRs, together with normal tympanometry and the presence of OAE, were classical features of auditory neuropathy. Absent ABRs suggested that the defect localizes to the synapse between the hair cells and the auditory nerve. As imaging studies revealed partial atrophy of the vestibulocochlear nerve, their vestibular and cochlear components and the patients manifested peripheral neuropathy, we assume that the disease process affects the vestibulocochlear nerve fibers, particularly their distal parts. It should be also taken into account that absent ABRs may represent a progressed state of the disease, which has begun in the proximal part of the vestibulocochlear nerve as it is observed in other hereditary neurological diseases affecting mitochondrial function such as Friedreich's ataxia or Charcot-Marie-Tooth (excluded in the proband based on genetic tests) $[54,55]$.

Partial atrophy of the vestibular nerves was less marked than the atrophy of the cochlear nerve and functionally sufficient to provide normal results of the vestibulo-ocular reflex in caloric tests and to some extend of the rotational testing. However, the number of vestibular nerve fibers discharging synchronously appears insufficient to obtain normal results of VEMP responses. It should be also considered that abnormal or absent VEMPs could be a consequence of the neuropathological process affecting the medial vestibulospinal tract (VEMP descending pathway) as diminished cervical enlargement was found in both patients. Asymptomatic vestibular disorders are commonly observed in patients with auditory neuropathy accompanied by peripheral neuropathy [56].

\section{Conclusions}

Our study presents a detailed and systematic assessment of the auditory and nervous systems in patients with PRLTS5, which sheds new insight on the phenotype and disease process of the mitochondrial disorder. We show for the first time in PRLTS patients (1) a complex mechanism of hearing impairment, comprising of cochlear dysfunction and dyssynchronous auditory nerve function, (2) partial atrophy of the vestibulocochlear nerves and their auditory and vestibular parts and (3) aberrant proportion of the cerebellum white and grey matters. Genetic workup provided further evidence on the causative role of TWNK mutations. From the seven known TWNK mutations underlying PRLTS5 one has recurred in our patients and its detection in the second individual with PRLTS5 confirms the pathogenic potential of the variant. The second TWNK mutation has not been reported in the context of any disease and its identification represents a novel genetic association with PRLTS5.

\section{Additional file}

Additional file 1: Figure S1. Sequence-to-structure alignment between the human Twinkle protein ( $\mathrm{Hs}$ ) and the template (PDB code 1e0j). The numbers of residues that are not shown are specified in parentheses. Identical residues are highlighted in yellow. Locations of observed (for 1e0j) and predicted (for $\mathrm{Hs}$ ) secondary structure elements are marked above and below the corresponding sequences. Helices are presented as blue cylinders, while beta strands as orange arrows.

\section{Abbreviations}

PRLTS: Perrault syndrome; SNHL: bilateral sensorineural hearing loss; MRI: magnetic resonance imaging; TWNK: twinkle mtDNA helicase; mtDNA: mitochondrial DNA; WES: whole-exome sequencing; EMG: electromyography; ABRs: auditory brainstem responses; GS/MS: gas chromatography-mass spectrometry; LC-MS/MS: liquid chromatography-tandem mass spectrometry; SDFS: spinocerebellar degeneration functional score; OAE: otoacoustic emissions; $\mathrm{nHL}$ : normal hearing level; cVEMP: cervical vestibular evoked myogenic potentials; OVEMP: ocular vestibular evoked myogenic potentials; MTDPS7: mitochondrial DNA depletion syndrome 7; IOSCA: infantile-onset spinocerebellar ataxia; PEOA3: autosomal dominant progressive external ophthalmoplegia.

\section{Authors' contributions}

$\mathrm{MO}$ analyzed the data and wrote the manuscript; $\mathrm{DO}, \mathrm{AP}, \mathrm{UL}$ performed genotyping and analyzed the data; MO, IS, GT, KK, TW, MF, HS participated in phenotyping and clinical data collection; DO, MŁ, DP, RP performed computational analysis; RP performed statistical analysis and participated in discussion; All authors read and approved the final manuscript.

\section{Author details \\ ${ }^{1}$ Department of Genetics, World Hearing Center, Institute of Physiology and Pathology of Hearing, Mokra 17, Kajetany/Warsaw, 05-830 Nadarzyn, Poland. ${ }^{2}$ Laboratory of Functional and Structural Genomics, Centre of New Technologies, University of Warsaw, Warsaw, Poland. ${ }^{3}$ Department of Experi- mental Audiology, Institute of Physiology and Pathology of Hearing, Kajetany/ Warsaw, Poland. ${ }^{4}$ Bioimaging Research Center, Institute of Physiology and Pathology of Hearing, Kajetany/Warsaw, Poland. ${ }^{5}$ Department of Otoneu- rology, Institute of Physiology and Pathology of Hearing, Kajetany/Warsaw, Poland. ${ }^{6}$ Department of Medical Genetics, Medical University of Warsaw, Warsaw, Poland. ${ }^{7}$ Oto-Rhino-Laryngology Surgery Clinic, Institute of Physiol- ogy and Pathology of Hearing, Kajetany/Warsaw, Poland.}

\section{Acknowledgements}

Not applicable.

\section{Competing interests}

The authors declare that they have no competing interests.

\section{Availability of data and materials}

Please contact author for data requests.

\section{Consent for publication}

A consent for publication has been obtained.

\section{Ethics approval and consent to participate}

The study was approved by the bioethics committee at the Institute of Physiology and Pathology of Hearing and performed according to the Declaration of Helsinki. Written informed consent was obtained from each participant.

\section{Funding}

This work was funded by the Institute of Physiology and Pathology of Hearing and Grants of the Polish National Science Centre 2011/03/D/NZ5/05592,

2014/15/B/ST6/05082 and 2013/09/B/NZ2/00121.

Received: 28 October 2016 Accepted: 25 January 2017

Published online: 08 February 2017 


\section{References}

1. Newman WG, Friedman TB, Conway GS. Perrault Syndrome. 1993.

2. Rehman $A U$, Friedman TB, Griffith AJ. Unresolved questions regarding human hereditary deafness. Oral Dis. 2016. doi:10.1111/odi.12516.

3. Gispert S, Parganlija D, Klinkenberg M, Drose S, Wittig I, Mittelbronn M, Grzmil P, Koob S, Hamann A, Walter M, et al. Loss of mitochondrial peptidase Clpp leads to infertility, hearing loss plus growth retardation via accumulation of CLPX, mtDNA and inflammatory factors. Hum Mol Genet. 2013;22:4871-87

4. Pierce SB, Chisholm KM, Lynch ED, Lee MK, Walsh T, Opitz JM, Li W, Klevit RE, King MC. Mutations in mitochondrial histidyl tRNA synthetase HARS2 cause ovarian dysgenesis and sensorineural hearing loss of Perrault syndrome. Proc Natl Acad Sci USA. 2011;108:6543-8.

5. Jenkinson EM, Rehman AU, Walsh T, Clayton-Smith J, Lee K, Morell RJ, Drummond MC, Khan SN, Naeem MA, Rauf B, et al. Perrault syndrome is caused by recessive mutations in CLPP, encoding a mitochondrial ATPdependent chambered protease. Am J Hum Genet. 2013;92:605-13.

6. Morino H, Pierce SB, Matsuda Y, Walsh T, Ohsawa R, Newby M, HirakiKamon K, Kuramochi M, Lee MK, Klevit RE, et al. Mutations in Twinkle primase-helicase cause Perrault syndrome with neurologic features. Neurology. 2014;83:2054-61.

7. Fiumara A, Sorge G, Toscano A, Parano E, Pavone L, Opitz JM. Perrault syndrome: evidence for progressive nervous system involvement. Am J Med Genet A. 2004;128A:246-9.

8. Kobe C, Kracht LW, Timmermann L, Bachmann J, Schmidt MC. Perrault syndrome with progressive nervous system involvement. Clin Nucl Med. 2008;33:922-4.

9. Pierce SB, Walsh T, Chisholm KM, Lee MK, Thornton AM, Fiumara A, Opitz JM, Levy-Lahad E, Klevit RE, King MC. Mutations in the DBPdeficiency protein HSD17B4 cause ovarian dysgenesis, hearing loss, and ataxia of Perrault syndrome. Am J Hum Genet. 2010;87:282-8.

10. Demain LA, Urquhart JE, O'Sullivan J, Williams SG, Bhaskar SS, Jenkinson EM, Lourenco CM, Heiberg A, Pearce SH, Shalev SA, et al. Expanding the genotypic spectrum of Perrault syndrome. Clin Genet. 2016. doi:10.1111/cge.12776.

11. McMillan HJ, Worthylake T, Schwartzentruber J, Gottlieb CC, Lawrence SE, Mackenzie A, Beaulieu CL, Mooyer PA, Wanders RJ, Majewski J, et al. Specific combination of compound heterozygous mutations in 17beta-hydroxysteroid dehydrogenase type 4 (HSD17B4) defines a new subtype of D-bifunctional protein deficiency. Orphanet J Rare Dis. 2012;7:90.

12. Lines MA, Jobling R, Brady L, Marshall CR, Scherer SW, Rodriguez AR, Lee L, Lang AE, Mestre TA, Wanders RJ, et al. Peroxisomal D-bifunctional protein deficiency: three adults diagnosed by whole-exome sequencing. Neurology. 2014;82:963-8.

13. Lerat J, Jonard L, Loundon N, Christin-Maitre S, Lacombe D, Goizet C, Rouzier C, Van Maldergem L, Gherbi S, Garabedian EN, et al. An application of NGS for molecular investigations in Perrault syndrome: study of 14 families and review of the literature. Hum Mutat. 2016;37:1354-62.

14. Pierce SB, Gersak K, Michaelson-Cohen R, Walsh T, Lee MK, Malach D, Klevit RE, King MC, Levy-Lahad E. Mutations in LARS2, encoding mitochondrial leucyl-tRNA synthetase, lead to premature ovarian failure and hearing loss in Perrault syndrome. Am J Hum Genet. 2013;92:614-20.

15. Solda G, Caccia S, Robusto M, Chiereghin C, Castorina P, Ambrosetti U, Duga S, Asselta R. First independent replication of the involvement of LARS2 in Perrault syndrome by whole-exome sequencing of an Italian family. J Hum Genet. 2016;61:295-300.

16. Ahmed S, Jelani M, Alrayes N, Mohamoud HS, Almramhi MM, Anshasi W, Ahmed NA, Wang J, Nasir J, Al-Aama JY. Exome analysis identified a novel missense mutation in the CLPP gene in a consanguineous Saudi family expanding the clinical spectrum of Perrault syndrome type-3. J Neurol Sci. 2015;353:149-54.

17. Dursun F, Mohamoud HS, Karim N, Naeem M, Jelani M, Kirmizibekmez $\mathrm{H}$. Novel missense mutation in the CLPP gene causes Perrault syndrome type-3 in a Turkish family. J Clin Res Pediatr Endocrinol. 2016. doi:10.4274/ jcrpe. 2717.

18. Spelbrink JN, Li FY, Tiranti V, Nikali K, Yuan QP, Tariq M, Wanrooij S, Garrido N, Comi G, Morandi L, et al. Human mitochondrial DNA deletions associated with mutations in the gene encoding Twinkle, a phage T7 gene 4-like protein localized in mitochondria. Nat Genet. 2001;28:223-31.
19. Fernandez-Millan P, Lazaro M, Cansiz-Arda S, Gerhold JM, Rajala N, Schmitz CA, Silva-Espina C, Gil D, Bernado P, Valle M, et al. The hexameric structure of the human mitochondrial replicative helicase Twinkle. Nucleic Acids Res. 2015:43:4284-95.

20. Anheim M, Fleury M, Monga B, Laugel V, Chaigne D, Rodier G, Ginglinger E, Boulay C, Courtois S, Drouot N, et al. Epidemiological, clinical, paraclinical and molecular study of a cohort of 102 patients affected with autosomal recessive progressive cerebellar ataxia from Alsace, Eastern France: implications for clinical management. Neurogenetics. 2010;11:1-12.

21. Jenike MA, Breiter HC, Baer L, Kennedy DN, Savage CR, Olivares MJ, O'Sullivan RL, Shera DM, Rauch SL, Keuthen N, et al. Cerebral structural abnormalities in obsessive-compulsive disorder. A quantitative morphometric magnetic resonance imaging study. Arch Gen Psychiatry. 1996;53:625-32.

22. Filipek PA, Richelme C, Kennedy DN, Caviness VS Jr. The young adult human brain: an MRI-based morphometric analysis. Cereb Cortex. 1994:4:344-60

23. Leonard CM, Towler S, Welcome S, Halderman LK, Otto R, Eckert MA, Chiarello C. Size matters: cerebral volume influences sex differences in neuroanatomy. Cereb Cortex. 2008;18:2920-31.

24. Audiology BSo. Recommended procedures for pure-tone audiometry using a manually operated instrument. Br J Audiol. 1981;15:213-6.

25. Piłka E. Testy słowne dostępne i wykorzystywane w Polsce w audiometrii mowy — rys historyczny. Nowa Audiofonologia. 2015:4:67-74.

26. Jerger J. Clinical experience with impedance audiometry. Arch Otolaryngol. 1970;92:311-24.

27. Jedrzejczak WW, Konopka W, Kochanek K, Skarzynski H. Otoacoustic emissions in newborns evoked by $0.5 \mathrm{kHz}$ tone bursts. Int J Pediatr Otorhinolaryngol. 2015;79:1522-6.

28. Jedrzejczak WW, Kochanek K, Trzaskowski B, Pilka E, Skarzynski PH, Skarzynski H. Tone-burst and click-evoked otoacoustic emissions in subjects with hearing loss above 0.25, 0.5, and $1 \mathrm{kHz}$. Ear Hear. 2012;33:757-67.

29. Jedrzejczak WW, Lorens A, Piotrowska A, Kochanek K, Skarzynski H. Otoacoustic emissions evoked by $0.5 \mathrm{kHz}$ tone bursts. J Acoust Soc Am. 2009;125:3158-65.

30. Kochanek KM, Sliwa L, Golebiowski M, Pilka A, Skarzynski H. Comparison of 3 ABR methods for diagnosis of retrocochlear hearing impairment. Med Sci Monit. 2015;21:3814-24.

31. Ploski R, Pollak A, Muller S, Franaszczyk M, Michalak E, Kosinska J, Stawinski P, Spiewak M, Seggewiss H, Bilinska ZT. Does p. Q247X in TRIM63 cause human hypertrophic cardiomyopathy? Circ Res. 2014;114:e2-5.

32. McKenna A, Hanna M, Banks E, Sivachenko A, Cibulskis K, Kernytsky A, Garimella K, Altshuler D, Gabriel S, Daly M, DePristo MA. The genome analysis toolkit: a MapReduce framework for analyzing next-generation DNA sequencing data. Genome Res. 2010;20:1297-303.

33. DePristo MA, Banks E, Poplin R, Garimella KV, Maguire JR, Hartl C, Philippakis AA, del Angel G, Rivas MA, Hanna M, et al. A framework for variation discovery and genotyping using next-generation DNA sequencing data. Nat Genet. 2011:43:491-8.

34. Robinson JT, Thorvaldsdottir H, Winckler W, Guttman M, Lander ES, Getz G, Mesirov JP. Integrative genomics viewer. Nat Biotechnol. 2011;29:24-6.

35. Adzhubei I, Jordan DM, Sunyaev SR. Predicting functional effect of human missense mutations using PolyPhen-2. Curr Protoc Hum Genet. 2013; Chapter 7:Unit7 20.

36. Kumar P, Henikoff S, Ng PC. Predicting the effects of coding non-synonymous variants on protein function using the SIFT algorithm. Nat Protoc. 2009:4:1073-81.

37. Schwarz JM, Cooper DN, Schuelke M, Seelow D. MutationTaster2: mutation prediction for the deep-sequencing age. Nat Methods. 2014:11:361-2

38. Altschul SF, Madden TL, Schaffer AA, Zhang J, Zhang Z, Miller W, Lipman DJ. Gapped BLAST and PSI-BLAST: a new generation of protein database search programs. Nucleic Acids Res. 1997;25:3389-402.

39. Li W, Godzik A. Cd-hit: a fast program for clustering and comparing large sets of protein or nucleotide sequences. Bioinformatics. 2006;22:1658-9.

40. Katoh K, Standley DM. MAFFT multiple sequence alignment software version 7: improvements in performance and usability. Mol Biol Evol. 2013;30:772-80.

41. Kurowski MA, Bujnicki JM. GeneSilico protein structure prediction metaserver. Nucleic Acids Res. 2003:31:3305-7. 
42. Ginalski K, Rychlewski L. Protein structure prediction of CASP5 comparative modeling and fold recognition targets using consensus alignment approach and 3D assessment. Proteins. 2003;53(Suppl 6):410-7.

43. Jaroszewski L, Li Z, Cai XH, Weber C, Godzik A. FFAS server: novel features and applications. Nucleic Acids Res. 2011;39:W38-44.

44. Soding J. Protein homology detection by HMM-HMM comparison. Bioinformatics. 2005;21:951-60.

45. Fiser A, Sali A. Modeller: generation and refinement of homology-based protein structure models. Methods Enzymol. 2003;374:461-91.

46. Wiederstein M, Sippl MJ. ProSA-web: interactive web service for the recognition of errors in three-dimensional structures of proteins. Nucleic Acids Res. 2007;35:W407-10.

47. McGuffin LJ, Bryson K, Jones DT. The PSIPRED protein structure prediction server. Bioinformatics. 2000;16:404-5.

48. Singleton MR, Sawaya MR, Ellenberger T, Wigley DB. Crystal structure of T7 gene 4 ring helicase indicates a mechanism for sequential hydrolysis of nucleotides. Cell. 2000;101:589-600.

49. Nikali K, Suomalainen A, Saharinen J, Kuokkanen M, Spelbrink JN, Lonnqvist T, Peltonen L. Infantile onset spinocerebellar ataxia is caused by recessive mutations in mitochondrial proteins Twinkle and Twinky. Hum Mol Genet. 2005;14:2981-90.
50. Hakonen AH, Isohanni P, Paetau A, Herva R, Suomalainen A, Lonnqvist T. Recessive Twinkle mutations in early onset encephalopathy with mtDNA depletion. Brain. 2007;130:3032-40.

51. Tyynismaa H, Mjosund KP, Wanrooij S, Lappalainen I, Ylikallio E, Jalanko A, Spelbrink JN, Paetau A, Suomalainen A. Mutant mitochondrial helicase Twinkle causes multiple mtDNA deletions and a late-onset mitochondrial disease in mice. Proc Natl Acad Sci USA. 2005;102:17687-92.

52. Goffart S, Cooper HM, Tyynismaa H, Wanrooij S, Suomalainen A, Spelbrink $J N$. Twinkle mutations associated with autosomal dominant progressive external ophthalmoplegia lead to impaired helicase function and in vivo mtDNA replication stalling. Hum Mol Genet. 2009;18:328-40.

53. King KA, Makishima T, Zalewski CK, Bakalov VK, Griffith AJ, Bondy CA, Brewer CC. Analysis of auditory phenotype and karyotype in 200 females with Turner syndrome. Ear Hear. 2007;28:831-41.

54. Rance G, Starr A. Pathophysiological mechanisms and functional hearing consequences of auditory neuropathy. Brain. 2015;138:3141-58.

55. Cacace AT, Pinheiro JM. The mitochondrial connection in auditory neuropathy. Audiol Neurootol. 2011;16:398-413.

56. Fujikawa S, Starr A. Vestibular neuropathy accompanying auditory and peripheral neuropathies. Arch Otolaryngol Head Neck Surg. 2000;126:1453-6.

\section{Submit your next manuscript to BioMed Central and we will help you at every step:}

- We accept pre-submission inquiries

- Our selector tool helps you to find the most relevant journal

- We provide round the clock customer support

- Convenient online submission

- Thorough peer review

- Inclusion in PubMed and all major indexing services

- Maximum visibility for your research

Submit your manuscript at www.biomedcentral.com/submit 